\title{
Spin-singlet hierarchy in the fractional quantum Hall effect
}

\author{
Kazusumi Ino \\ Nomura Research Institute, Hongo 2-2-9, Bunkyo-ku, Tokyo, 113-0033, Japan
}

\begin{abstract}
We show that the so-called permanent quantum Hall states are formed by the integer quantum Hall effects on the Haldane-Rezayi quantum Hall state. Novel conformal field theory description along with this picture is deduced. The odd denominator plateaux observed around $\nu=\frac{5}{2}$ are the permanent states if the $\nu=\frac{5}{2}$ plateau is the Haldane-Rezayi state. We point out that there is no such hierarchy on other candidate states for $\nu=\frac{5}{2}$. We propose experiments to test our prediction. PACS: $73.40 \mathrm{Hm}, 74.20-\mathrm{z}, 11.25 . \mathrm{Hf}$
\end{abstract}

In 1987, Willet et al discovered a quantum Hall effect at an even-denominator filling fraction $\nu=5 / 2$ [1]. This is so far the only even-denominator Fractional Quantum Hall Effect (FQHE) found in a single-layer system. The tilted field experiments suggest that the plateau is a spinunpolarized singlet state [2]. This motivated Haldane and Rezayi to propose a variational ansatz for the spinsinglet ground state, the Haldane-Rezayi (HR) state [3]. Although it has satisfactory features as a candidate for the $\nu=5 / 2$ plateau, it was shown that the hollow-core Hamiltionian with which the HR state is the exact ground state does not reproduce the realistic Hamiltonian even in the second Landau level [4. Recent numerical studies [5] show that the ground state may be a spin-polarized pfaffian-like state [6]. If the state is spin-polarized, however, new explanation for the results of tilted field experiments is required. Some suggestions, such as the effect of thickness of real sample have been made. However, the discussions are not satisfactory yet and the final answer remains to be seen.

The enigmatic $\nu=5 / 2$ plateau is a FQHE at the second Landau level. In the FQHEs in the lowest Landau level, the observed plateaux are well described by the Jain's hierarchy [7]. The key of Jain's idea is the non-perturbative object called composite fermion. It is formed by the electron and the vortex. At $\nu=1 / 2$, the external magnetic field is completely screened by the formation of composite fermions and they form an unusual metallic state ; a Fermi liquid of composite fermions [8]. The Jain's picture is that the FQHEs are the integer QHEs in the vicinity of the filling fraction $\nu=1 / 2$. The Landau levels emerging here are those for composite fermion. This scheme predicts most of the observed FQH plateaux around $\nu=1 / 2$.

It is worth while asking whether similar scheme holds in the second Landau level. It seems natural to consider that the $\nu=5 / 2$ plateau might have the same role in the FQHE at the second Landau level as the $\nu=1 / 2$ state does in the FQHE at the lowest Landau level. Suppose that the Fermi surface of the Fermi liquid of composite fermions has an instability to form a pair condensate. Even in this case, the Landau levels are formed by adding magnetic field and QH plateaux would appear. These plateaux are not expected to be the ordinary FQH state : they should inherit the feature of the state at $\nu=5 / 2$ such as pairing of composite fermions. It suggests that the odd-denominator plateaux around $\nu=5 / 2$ $(\nu=7 / 3,8 / 3 \ldots)$ observed in Ref. [1] would also be a part of the enigma.

In this paper, we will give evidences that this will be the case. We show that a class of spin-singlet QH states, namely the so-called permanent $\mathrm{QH}$ states (which are variants of the HR state first proposed in Ref. [9]) are formed by integer QH effects around the HR state. This observation is supported by the existence of conformal field theory (CFT) description of novel kind which we deduce below. Based on this novel description, we develop a hierarchical scheme of the permanent $\mathrm{QH}$ states. It yields a candidate comprehensive scheme for FQH plateaux $\nu=7 / 3,8 / 3 \ldots$ etc around $\nu=5 / 2$. We also point out that such a construction only exists for the HR state i.e. no such hierarchy for Pfaffian or other candidate states for the $\nu=5 / 2$ plateau exists. If we believe in the validity of the idea of hierarchy around $\nu=5 / 2$, the existence of the construction gives an evidence that the $\nu=5 / 2$ plateau is the HR state.

First we note that Bonesteel recently showed that composite fermions without additional degrees of freedom do not form a pair condensate by themselves [10] based on the celebrated theory of Ref. [8]. Thus the essential ingredient which should be studied is the degrees of freedom which drive composite fermions to form a pair.

Let us consider the relation between the simplest permanent state and the HR state. The wave function of the permanent state at $\nu=\frac{1}{q}=\frac{1}{p+1}(q=p+1, p$ : even integer) has a simple form, $\Psi_{\text {per }}=$

$$
\operatorname{per}\left(\frac{1}{z_{i}^{\uparrow}-z_{j}^{\downarrow}}\right) \prod\left(z_{i}-z_{j}\right)^{q} \exp \left(-\frac{1}{4} \sum_{i}^{N}|z|^{2}\right),
$$

where $N$ is the number of electrons. The HR state at $\nu=\frac{1}{p}(p=2$ for $\nu=5 / 2=2+1 / 2)$ has a more involved form (we omit the exponential factor hereafter), $\Psi_{\mathrm{HR}}=$

$\operatorname{per}\left(\frac{1}{z_{i}^{\uparrow}-z_{j}^{\downarrow}}\right) \prod\left(z_{i}^{\uparrow}-z_{j}^{\downarrow}\right)^{q-2} \prod\left(z_{i}^{\uparrow}-z_{j}^{\uparrow}\right)^{q} \prod\left(z_{i}^{\downarrow}-z_{j}^{\downarrow}\right)^{q}$.

From these forms, it seems that the HR state can be realized by some additional degrees of freedom and structure 
on $\Psi_{\text {per. }}$. However the HR state also has the following form:

$$
\Psi_{\mathrm{HR}}=\operatorname{det}\left(\frac{1}{\left(z_{i}^{\uparrow}-z_{j}^{\downarrow}\right)^{2}}\right) \prod\left(z_{i}-z_{j}\right)^{p}
$$

It is known that the permanent factor in (1i) and the determinant in (3) have a simple relation [3]

$\operatorname{det}\left(\frac{1}{\left(z_{i}^{\uparrow}-z_{j}^{\downarrow}\right)^{2}}\right)=\operatorname{per}\left(\frac{1}{z_{i}^{\uparrow}-z_{j}^{\downarrow}}\right) \operatorname{det}\left(\frac{1}{z_{i}^{\uparrow}-z_{j}^{\downarrow}}\right)$.

This can be rewritten as

$\operatorname{per}\left(\frac{1}{z_{i}^{\uparrow}-z_{j}^{\downarrow}}\right)=\operatorname{det}\left(\frac{1}{\left(z_{i}^{\uparrow}-z_{j}^{\downarrow}\right)^{2}}\right) \operatorname{det}\left(\frac{1}{z_{i}^{\uparrow}-z_{j}^{\downarrow}}\right)^{-1}$.

This shows that the wave function of the permanent state can be written as

$$
\Psi_{\text {per }}=\left[\operatorname{det}\left(\frac{1}{z_{i}^{\uparrow}-z_{j}^{\downarrow}}\right)^{-1} \prod\left(z_{i}-z_{j}\right)\right] \Psi_{\mathrm{HR}} .
$$

This factorization is just the reminiscent of the one for the Laughlin wave function in the Jain's paper [7]. The Langhlin factor in (6) implies the formation of the filled Landau level i.e. the $\nu=1$ integer $\mathrm{QH}$ effect. The factor of the inverse of determinant is a novel one and requires an interpretation.

In general, the composite fermions in the Landau level repulsively interacts each other by the Coulomb interaction. This gives rise to the Laughlin factor. When the composite fermions are paired, it would also have an effect on the strength of pairing. As $\operatorname{det}\left(\frac{1}{z_{i}^{\uparrow}-z_{j}^{\downarrow}}\right)$ has poles of order one, $\operatorname{det}\left(\frac{1}{z_{i}^{\uparrow}-z_{j}^{\downarrow}}\right)^{-1}$ has zeroes of order one for each pair. Thus it will reduce the strength of pairing by order one. The reduction is physically interpreted as the effect of the formation of the filled Landau level on the pairing.

Thus the permanent states can be seen as the integer QHEs around the HR state. Extension to multiple filled Landau levels is straight forward.

As it turns out, this observation realizes in the CFT description. As observed in [6], the pairing part of the permanent state can be written as the correlator of the $c=-1$ bosonic ghosts $\beta-\gamma$ [11], whose conformal dimensions $\Delta$ are $1 / 2$ (we also denote them as $\beta^{\alpha}, \quad \alpha=\uparrow, \downarrow$ ) :

$$
\operatorname{per}\left(\frac{1}{z_{i}^{\uparrow}-z_{j}^{\downarrow}}\right)=\left\langle\prod_{i=1}^{N / 2} \beta\left(z_{i}^{\uparrow}\right) \gamma\left(z_{i}^{\downarrow}\right)\right\rangle .
$$

In terms of these fields and a chiral boson $\varphi$ $\left(\left\langle\varphi\left(z_{1}\right) \varphi\left(z_{2}\right)\right\rangle=-\log \left(z_{1}-z_{2}\right)\right)$, the electrons in the permanent state are represented by $\beta^{\alpha} e^{i \sqrt{q} \varphi}$. There is an ingenious bosonization for the $\beta-\gamma$ system [11, in which $\beta$ and $\gamma$ are bosonized into $c=-2 \xi-\eta$ system and a chiral boson $\phi$ with negative signature as follows:

$$
\beta=\partial \xi e^{\phi}, \quad \gamma=\eta e^{-\phi} .
$$

(8) reproduces the formula (四). Note that the zero mode of $\xi$ does not appear in this bosonization. As in [11], the twisted sector is usually created by the spin field $\Sigma=e^{\phi / 2}$ of $\Delta=-1 / 8$. By this field, the elementary quasiholes in the permanent state is given by $\Sigma e^{\frac{1}{2 \sqrt{q}} \varphi}$. As noted in [12], the twisted sector is infinitely degenerated, due to the appearance of the zero mode of $\beta^{\alpha}$.

Although one can construct the bulk wave functions by this system, it does not necessarily imply that it is the right description of the permanent state. One way to check it is to consider the consistency of the edge theory which governs the low energy physics at the thermodynamic limit $N \rightarrow \infty$. The edge excitations are generated by the $\beta-\gamma$ system and $i \sqrt{\nu} \partial \varphi$. The excitations on each sector contribute to the partition function through the Virasoro characters :

$$
\begin{aligned}
\chi_{1} & =\frac{1}{2}\left(\frac{\eta(t)}{\vartheta_{3}(t)}+\frac{\eta(t)}{\vartheta_{2}(t)}\right), \\
\chi_{\beta} & =\frac{1}{2}\left(\frac{\eta(t)}{\vartheta_{3}(t)}-\frac{\eta(t)}{\vartheta_{2}(t)}\right), \\
\chi_{\Sigma} & =\frac{\eta(t)}{\vartheta_{4}(t)},
\end{aligned}
$$

where $t$ is $-\frac{1}{\tau}$ ( $\tau$ is the modular parameter). In the twisted sector the degenerated ground states are summed up and regularized [12, 13]. The characters for the $\mathrm{U}(1)$ sector are given by $\chi_{r / q}(t)=\frac{1}{\sqrt{q}} \frac{\vartheta_{3}\left(\frac{r}{q}\left(\frac{t}{q}\right)\right.}{\eta(t)}, \chi_{r / q}^{\vee}(t)=$ $\frac{1}{\sqrt{q}} \frac{\vartheta_{2}\left(\frac{r}{q} \mid \frac{t}{q}\right)}{\eta(t)}$ and $\chi_{(r+1 / 2) / q}(t)=\frac{1}{\sqrt{q}} \frac{\vartheta_{4}\left(\frac{r}{q} \mid \frac{t}{q}\right)}{\eta(t)}$. To check the consistency, we consider the edge theory for the state on the cylinder. The edge states on two edges are divided into finite sectors up to the electrons, and the partition function has a corresponding factorization. Eventually we end up with three terms:

$$
\begin{gathered}
V=\frac{1}{2 q} \sum_{r=1}^{q}\left|\frac{\vartheta_{3}\left(\frac{r}{q} \mid \frac{t}{q}\right)}{\vartheta_{3}(t)}\right|^{2}, \\
W=\frac{1}{2 q} \sum_{r=1}^{q}\left|\frac{\vartheta_{2}\left(\frac{r}{q} \mid \frac{t}{q}\right)}{\vartheta_{2}(t)}\right|^{2}, \\
X=\frac{1}{q} \sum_{r=1}^{q}\left|\frac{\vartheta_{4}\left(\frac{r}{q} \mid \frac{t}{q}\right)}{\vartheta_{4}(t)}\right|^{2} .
\end{gathered}
$$

The problem here is that the multiplicities of the last two characters $V, W$ and $X$ do not match, thus do not satisfy a necessary condition for the modular invariance [14]. To achieve the matching, we need additional zero modes which give additional degeneracy to $V$ and $W$. One expect that the zero mode $\xi$ may play a role like in the picture changing operation of superstring theories 
[11. However, naive addition of $\xi$ will add the factor 2 to $V, W$ and $X$ equally.

We note that these factors 2 from $\xi$ arises since we create the twisted sector by $e^{\phi / 2}$. Actually this is not the only possibility; one can instead twist the $c=-2$ part. Twisiting the $c=-2$ part will maximally extend the $\xi-\eta$ system to the symplectic fermions' 17. We thus should deal with the symplectic fermions $\theta^{\uparrow}, \theta^{\downarrow}$. Now $\beta$ and $\gamma$ are bosonized into

$$
\beta=\partial \theta^{\uparrow} e^{\phi}, \quad \gamma=\partial \theta^{\downarrow} e^{-\phi} .
$$

The twisted sector of these fields is created by $\sigma$, the spin field for the symplectic fermions. The operator product of the spin fields is not closed within the $\beta-\gamma$ system, and the zero modes of $\theta$ naturally appear for consistency [17]. Thus this system is the extended $\beta-\gamma$ system. By virtue of the twisting we take, the zero modes of $\theta$ do not appear in the twisted sector, just leading to the multiplicities required above. The partition function $Z=2 V+2 W+X$ is modular invariant. The five sectors of the partition function correspond to the fields $1, \beta^{\alpha}, \sigma, \widetilde{I}(=\theta \bar{\theta})$ and $\beta^{\alpha} \widetilde{I}\left(\theta \bar{\theta}=\frac{1}{2}\left(\theta^{\uparrow} \theta^{\downarrow}-\theta^{\downarrow} \theta^{\uparrow}\right)\right.$.) Accordingly the permanent state on the torus have $5 q$ degeneracy. The torus wave functions are obtained as the conformal blocks using the torus version of the bosonization (15).

The conformal field theory we end up with supports our observation that the permanent states are formed by the integer $\mathrm{QH}$ effects around the HR state. First the electron fields are represented by the same degrees of freedom (symplectic fermions) of the electron fields in the HR state [15, 16, 18], together with additional chiral boson $\phi$ which is regarded as describing the effect of the formation of the filled Laudau level on the pairing. The quasiholes are also constructed from $\sigma$ which comes from the quasiholes of the HR state. The appearance of the zero modes of $\theta$, thus the presence of the logarithmic field $\widetilde{I}$ is also the reminiscent of the HR state. This implies that, in the formation of the permanent state, these zero modes from the d-wave pairing in the HR state survive.

As shown in [18], the edge state of the HR state has an instability to generate a flux quantized to half integer. The permanent state does not inherit this feature. The flux which couples to the spin is ill-defined in this case, so we can consider the Aharanov-Bohm flux. The partition function with the Aharanov-Bohm flux $Z(\Phi)$ has its minima for integer values of flux. This feature is ascribed to the fact that the $c=-1$ part of the permanent state has no background charge [1] while the $c=-2$ part of the HR state does.

The unitarity of the edge theory also is realized differently from the HR state. In the HR state, the unitarity is restored by the presence of spontaneous flux [18]. This exchanges the couplings between the pairing and the charge degrees of freedom, and the edge state becomes identical to the 331 state with the $c=1$ chiral Weyl fermion as its pairing degrees of freedom. On the other hand, we don't have such spontaneous flux in the permanent state. However, it is known that the $c=-1$ theory generates the Verma modules of the $c=2$ Virasoro algebra realized on the $\mathbf{Z}_{2}$ orbifold of complex boson [13. Thus the edge excitations of the permanent state can be viewed as generated by the $c=2$ Virasoro algebra and the unitarity is realized. Note that it differs from the relation between the $c=-2$ theory and the $c=1$ theory underlying the HR state, since the $c=-1$ theory only generates the Verma modules of the $c=2$ Virasoro algebra and the correspondence is not extended to the one between the solitonic sectors of two theories.

The construction we have considered can be extended to form hierarchy. This is possible since the permanent state has an odd denominator filling fraction. A few examples was given in 9, but no systematic construction has been given. Actually this is readily done in the following way. In general, the degrees of freedom of $m$ filled Landau levels are described by $m$ species of chiral bosons $\varphi_{i}(i=1, \cdots, m)$. The pairing part of the permanent state is expected to be unaffected by the formation of the multi Landau levels of composite fermion. The couplings between $\varphi_{i}$ are specified by an integer matrix $K$ [19,20]. The permanent analog of the Jain's hierarchy is obtained by taking $K$ as

$$
K_{a b}= \pm \delta_{a b}+s C_{a b},
$$

where $s$ is a positive even integer and $C_{a b}=1$ for $\forall a, b=1, \cdots, m$. The filling fraction is given by

$$
\nu=\frac{m}{m s \pm 1} .
$$

As shown in [20], there is an affine $\mathrm{SU}(m)$ symmetry of level $1 \mathrm{in}$ the $m$-th hierarchy. The quasiholes form $m$ kinds of integrable representations of $\mathrm{SU}(m)_{1}$, which are labeled as $a=1, \cdots, m$. They correspond to $a$-th antisymmetric tensor of $\mathrm{SU}(m)$ respectively, which couples to charge $\frac{a}{m} \nu$ up to $Z \nu$. Thus the primary fields for the excitations on the permanent state are $1 V_{j}^{a} e^{i \frac{l m+a}{m \sqrt{q}} \varphi}, \beta^{\alpha} V_{j}^{a} e^{\frac{l m+a}{m \sqrt{q}} \varphi}, \sigma V_{j}^{a} e^{i \frac{l m+a}{2 m \sqrt{q}} \varphi}, \widetilde{I} V_{j}^{a} e^{i \frac{l m+a}{m \sqrt{q}} \varphi}$ $\beta \widetilde{I} V_{j}^{a} e^{i \frac{l m+a}{m \sqrt{q}} \varphi}$ where $l=0, \cdots,(m s \pm 1)-1, q=1 / \nu$ and $V_{j}^{a}$ are the primary fields for the representation $a$ respectively. This completes our description of spin-singlet hierarchical structure formed on the HR state. We can similarly consider other hierarchical schemes which involve $\mathrm{SO}(m)$ or $\mathrm{Sp}(m)$ affine symmetry. It is interesting that there is also a Lorentzian affine symmetry by the presence of $\phi$.

We'd like to point out that the Pfaffian or other candidate states for the $\nu=5 / 2$ plateau cannot have such hierarchical structure on them. It will simply violate the Fock condition i.e. once we consider such a hierarchy, the wave functions we obtain cannot represent the electron system. Thus, if one assumes the validity of the idea of hierarchy around $\nu=5 / 2$, the existence of hierarchy on the HR state gives an evidence that the $\nu=5 / 2$ plateau is the HR state, and that the odd-denominator plateaux 
around it $(\nu=7 / 3,8 / 3 \ldots)$ are the hierarchical permanent $\mathrm{QH}$ states.

The major experimental consequence of our result is that tilted field experiments at these odd-denominator plateaux must behave similarly as the $\nu=5 / 2$ plateau [2] i.e. as spin-singlet. Unfortunately since, as far as we know, the experimental works so far have been focussed on the even-denominator FQH state, we don't have information to confirm this at present. Also the present experimental results are not enough to determine which hierarchical scheme is preferred around $\nu=5 / 2$, but we expect that the permanent analog of the Jain's hierarchy we constructed above may be most promising.

In this paper, we showed that the permanent QH states are formed by the integer QHEs on the HR state. Novel conformal field theory (the extended $\beta-\gamma$ system) description was deduced from the modular invariance condition of the edge states for the state on the cylinder (see also 21]). We also developed a hierarchical scheme of the permanent $\mathrm{QH}$ states, including the permanent analog of the Jain's hierarchy. It yields a candidate scheme for understanding the FQHEs around $\nu=5 / 2$. The major experimetal consequence is that tilted field experiments at the odd-denominator plateaux around $\nu=5 / 2$ will show the spin-singlet behavior as the enigmatic $\nu=5 / 2$ plateau. We propose experiments to test our prediction.

Acknowledgement The author would like to thank M. Kohmoto and H.Kuriki for useful discussions and M.Yamanaka for comments on the manuscript.
[1] R.L.Willet, J.P. Eisenstein, H.L. Stormer, D.C.Tsui, A.C. Gossard and J.H. English, Phys. Rev. Lett. 59 (1987) 1776.

[2] J.P. Eisenstein et al., Phys. Rev. Lett. 61 (1988) 997; J.P. Eisenstein et al. Surf.Sci.229(1990)31.

[3] F.D.M. Haldane and E.H. Rezayi, Phys. Rev. Lett. 60 (1988) 956 : ibid 1886.

[4] A.H. MacDonald, D.Yoshioka and S.M. Girvin, Phys. Rev. B39 (1989) 1932.

[5] R.H.Morf, Phys. Rev. Lett. 80(1998) 1505.

[6] G. Moore and N. Read, Nucl. Phys. B360 (1991) 362.

[7] J.K. Jain, Phys. Rev. Lett. 63,(1989) 199; Phys. Rev. B40(1989) 8079; Phys. Rev. B41(1990) 7653.

[8] B.I.Haperin, P.A.Lee and N.Read, Phys. Rev. B47(1992) 7312.

[9] D. Yoshioka, A.H. MacDonald and S.M.Girvin, Phys. Rev. B38 (1989) 3636.

[10] N.E.Bonesteel, Phys. Rev. Lett. 82(1999) 984.

[11] D. Friedan, E. Martinec and S.Shenker, Nucl. Phys. B271 (1986) 93 : see also D. Friedan, S. Shenker and E. Martinec, Phys. Lett. 160B(1985) 55; V.Knizhnik, Phys. Lett. 160B(1985) 403.

[12] N. Read and E.H. Rezayi, Phys. Rev. B54(1996) 16864.

[13] S. Guruswamy and A.W.W. Ludwig, Nucl. Phys. B519(1998) 661.

[14] A. Cappelli and G.R. Zemba, Nucl. Phys. B490 (1997) 595.

[15] M.Milovanović and N.Read, Phys. Rev. B53(1996) 13559.

[16] V. Gurarie, M. Flohr and C. Nayak, Nucl. Phys. B498 (1997) 513.

[17] K.Kausch, Curiosities at $c=-2$, hep-th/9510149.

[18] K. Ino, to appear in Phys. Rev. Lett. 82(1999).

[19] N.Read, Phys. Rev. Lett. 65 (1990) 1502.

[20] J. Fröhlich and A. Zee, Nucl. Phys. B364 (1 991)517.

[21] K. Ino, On the conformal field theory of the permanent quantum Hall states, in preparation. 